Supporting information for:

\title{
Kinetic Study on the Preparation of Fumaric Acid from Maleic Acid by Batch Noncatalytic Isomerization
}

Wangmi Chen ${ }^{a}$, Xiaoting Chen ${ }^{b}$, Shouzhi $Y^{b *}$

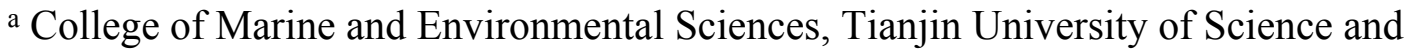

Technology, Tianjin 300457, China

${ }^{\mathrm{b}}$ College of Chemical Engineering and Materials Science, Tianjin University of Science and Technology, Tianjin 300457, China. 


\section{Contents}

1. (a) Typical appearance of the products. (b) SEM image of the products. S3

2. ${ }^{1} \mathrm{H}-\mathrm{NMR}$ spectrum of the products $\quad$ S3-S4

3. The analytical method of solution of the system of ordinary differential equations given by Equations (4) to (6)

S4-S7 
1. (a) Typical appearance of the products. (b) SEM image of the products.

(a)

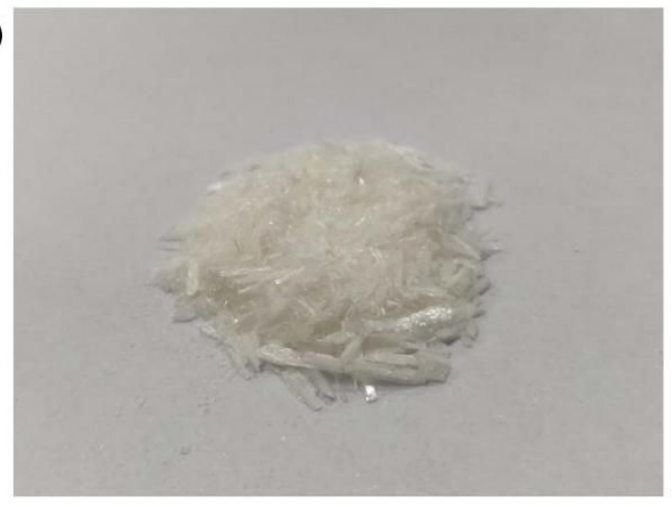

Figure S1. (a) Typical appearance of the products (b)

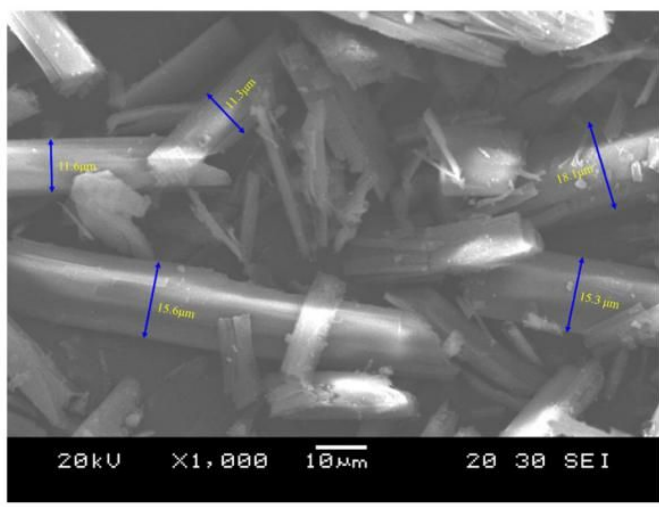

The mutual conversions of organic acids were simply accomplished by hydrothermal method, and white particle samples were obtained after washing with water (Figure 1a). The general morphology of the products obtained and purified after the selective conversion was characterized by SEM. The SEM image Figure $1 \mathrm{~b}$ showed that the products were obtained in a rod-like morphology, the diameters and lengths of which were in the range of $11-18 \mu \mathrm{m}$ and $60-105 \mu \mathrm{m}$, respectively. At the same time, there was no impurity could be found.

\section{2. ${ }^{1} \mathrm{H}-\mathrm{NMR}$ spectrum of the products}

The peak positions of the solid powder products were $13.124 \mathrm{ppm}, 6.638 \mathrm{ppm}, 2.517$ ppm, respectively. The peaks at $13.124 \mathrm{ppm}$ and $6.638 \mathrm{ppm}$ of the four peaks are characteristic peaks of the fumaric acid structure, while the peak at $2.517 \mathrm{ppm}$ is the fixed peak of the solvent DMSO. 


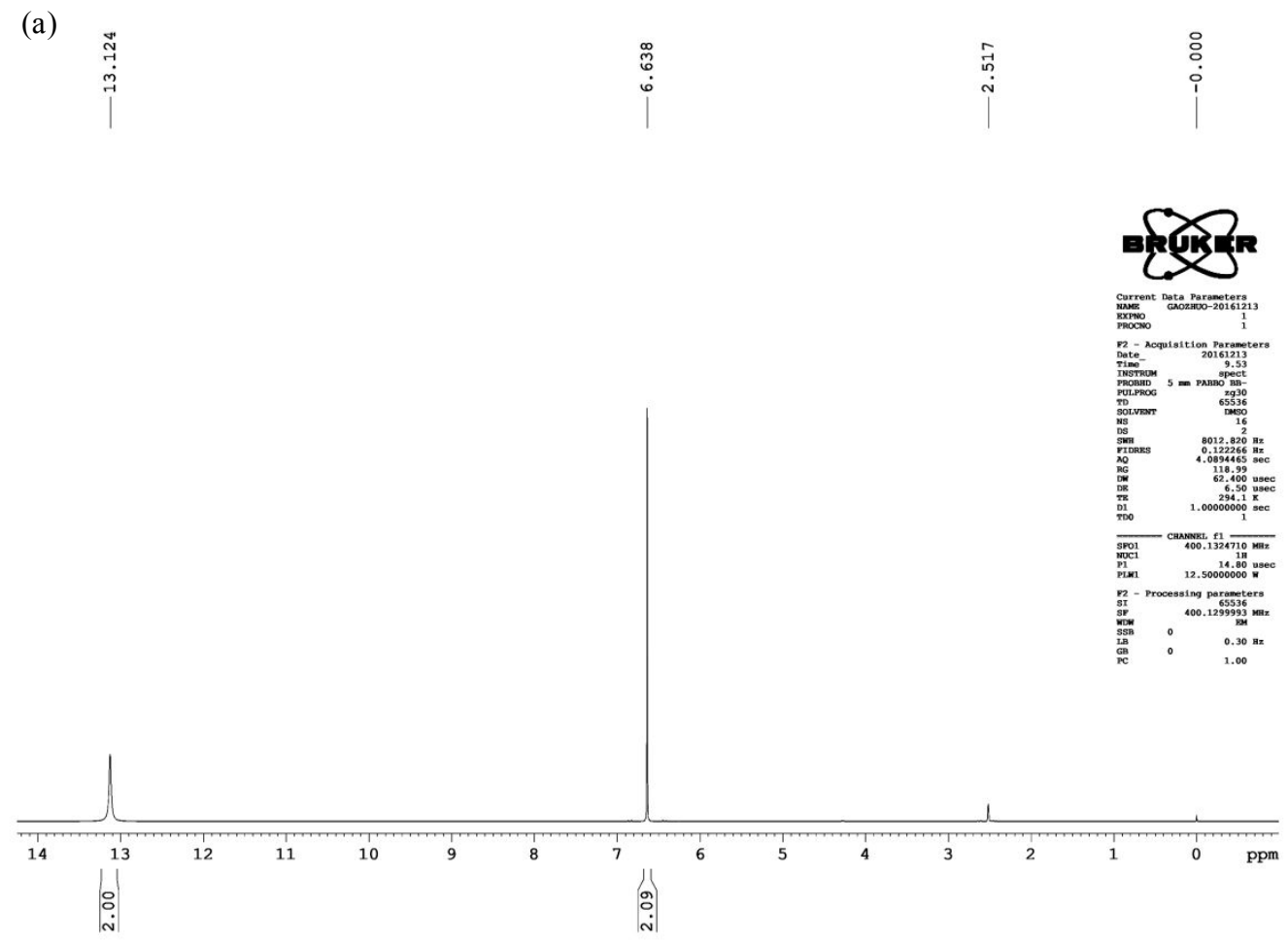

Figure S2. ${ }^{1} \mathrm{H}-\mathrm{NMR}$ spectrum of the products.

3. The analytical method of solution of the system of ordinary differential equations given by Equations (4) to (6)

To simplify the calculation, make the following assumptions.

$$
\begin{aligned}
& \mathrm{k}_{1}=\mathrm{a} \\
& {\mathrm{k}-{ }_{1}=\mathrm{b}}=\mathrm{k}_{2}=\mathrm{c} \\
& \mathrm{k}_{3}=\mathrm{e} \\
& \mathrm{k}_{-3}=\mathrm{f}
\end{aligned}
$$




$$
\begin{aligned}
& \left\{\begin{array}{l}
\frac{d x}{d t}=-(a+c) x+b y \\
\frac{d y}{d t}=a x-(b+f) y+e z \\
\frac{d z}{d t}=c x+f y-e z
\end{array}\right. \\
& g=x+y+z \\
& \left\{\begin{array}{l}
t=0 \\
x=g \\
y=0 \\
z=0
\end{array}\right. \\
& \left\{\frac{d x}{d t}=-(a+c) x+b y\right. \\
& \frac{d y}{d t}=a x-(b+f) y+e(g-x-y)=(a-e) x-(b+f+e) y+e g \\
& b y=\frac{d x}{d t}+(a+c) x \\
& y=\frac{1}{b} \cdot\left[\frac{d x}{d t}+(a+c) x\right] \\
& \frac{d y}{d t}=\frac{1}{b} \cdot\left[\frac{d^{2} x}{d t^{2}}+(a+c) \frac{d x}{d t}\right] \\
& \frac{1}{b} \cdot\left[\frac{d^{2} x}{d t^{2}}+(a+c) \frac{d x}{d t}\right]=(a-e) x-(b+f+e) y+e g \\
& \frac{1}{b} \cdot \frac{d^{2} x}{d t^{2}}+\frac{a+c}{b} \cdot \frac{d x}{d t}+\frac{b+e+f}{b} \cdot \frac{d x}{d t}+\frac{(b+e+f)(a+c)}{b} \cdot x-(a-e) x-e g=0 \\
& \frac{d^{2} x}{d t^{2}}+(a+b+c+e+f) \frac{d x}{d t}+[(a+e+f)(a+c)-b(a-e)] x-b e g=0 \\
& \lambda^{2}+(a+b+c+e+f) \lambda+[(a+e+f)(a+c)-b(a-e)]=0 \\
& \lambda_{1,2}=\frac{-(a+b+c+e+f) \pm \sqrt{(a+b+c+e+f)^{2}-4[c(b+e+f)+a f+a e-b e]}}{2} \\
& \left\{\begin{array}{l}
x^{*}=A \\
x^{* \prime}=0 \\
x^{* \prime \prime}=0
\end{array}\right.
\end{aligned}
$$




$$
\begin{aligned}
& {[(a+e+f)(a+c)-b(a-e)] A=b e g} \\
& A=\frac{b e g}{b c+c e+c f+a f+a e-b e}=\frac{g}{(a+c)\left(\frac{b+f}{b e}\right)+\frac{c}{e}-1} \\
& x=C_{1} e^{\lambda_{1} t}+C_{2} e^{\lambda_{2} t}+\frac{g}{(a+c)\left(\frac{b+f}{b e}\right)+\frac{c}{e}-1} \\
& g=C_{1}+C_{2}+\frac{g}{(a+c)\left(\frac{b+f}{b e}\right)+\frac{c}{e}-1} \\
& C_{1}+C_{2}=g\left[1-\frac{g}{(a+c)\left(\frac{b+f}{b e}\right)+\frac{c}{e}-1}\right] \\
& b y=\frac{d x}{d t}+(a+c) x=C_{1} \lambda_{1} e^{\lambda_{1} t}+C_{2} \lambda_{2} e^{\lambda_{2} t}+(a+c)\left[C_{1} e^{\lambda_{1} t}+C_{2} e^{\lambda_{2} t}+\frac{g}{(a+c)\left(\frac{b+f}{b e}\right)+\frac{c}{e}-1}\right] \\
& y=\frac{C_{1}}{b}\left(a+c+\lambda_{1}\right) e^{\lambda_{1} t}+\frac{C_{2}}{b}\left(a+c+\lambda_{2}\right) e^{\lambda_{2} t}+\frac{(a+c) g}{(a+c)\left(\frac{b+f}{e}\right)+\frac{b c}{e}-b} \\
& \frac{C_{1}}{b}\left(a+c+\lambda_{1}\right)+\frac{C_{2}}{b}\left(a+c+\lambda_{2}\right)+\frac{(a+c) g}{(a+c)\left(\frac{b+f}{e}\right)+\frac{b c}{e}-b}=0 \\
& \text { [ } C_{1}+C_{2}=g\left[1-\frac{g}{(a+c)\left(\frac{b+f}{b e}\right)+\frac{c}{e}-1}\right] \\
& \therefore\left\{\begin{array}{l}
\frac{C_{1}}{b}\left(a+c+\lambda_{1}\right)+\frac{C_{2}}{b}\left(a+c+\lambda_{2}\right)+\frac{(a+c) g}{(a+c)\left(\frac{b+f}{e}\right)+\frac{b c}{e}-b}=0 \\
C_{1}+C_{2}-g\left[1-\frac{g}{(a+c)\left(\frac{b+f}{b e}\right)+\frac{c}{e}-1}\right]=0
\end{array}\right.
\end{aligned}
$$




$$
\begin{aligned}
& \frac{C_{1}}{b}\left(a+c+\lambda_{1}\right)+\frac{C_{2}}{b}\left(a+c+\lambda_{2}\right)+\frac{(a+c) g}{(a+c)\left(\frac{b+f}{e}\right)+\frac{b c}{e}-b} \\
& -\frac{\left(a+c+\lambda_{1}\right)}{b}\left\{C_{1}+C_{2}-g\left[1-\frac{g}{(a+c)\left(\frac{b+f}{b e}\right)+\frac{c}{e}-1}\right]\right\}=0 \\
& C_{2}=\frac{g\left\{\lambda_{1}\left[\frac{g}{(a+c)\left(\frac{b+f}{b e}\right)+\frac{c}{e}-1}-1\right]-(a+c)\right\}}{\lambda_{2}-\lambda_{1}} \\
& C_{1}=g\left[1-\frac{g}{(a+c)\left(\frac{b+f}{b e}\right)+\frac{c}{e}-1}\right]-C_{2} \\
& =g\left[1-\frac{g}{(a+c)\left(\frac{b+f}{b e}\right)+\frac{c}{e}-1}\right]-\frac{g\left\{\lambda_{1}\left[\frac{g}{(a+c)\left(\frac{b+f}{b e}\right)+\frac{c}{e}-1}-1\right]-(a+c)\right\}}{\lambda_{2}-\lambda_{1}} \\
& =\frac{g\left\{\lambda_{2}\left[1-\frac{g}{(a+c)\left(\frac{b+f}{b e}\right)+\frac{c}{e}-1}\right]+(a+c)\right\}}{\lambda_{2}-\lambda_{1}} \\
& z=g-x-y=-\frac{C_{1}}{b} e^{\lambda_{1} t}\left(a+b+c+\lambda_{1}\right)-\frac{C_{2}}{b} e^{\lambda_{2} t}\left(a+b+c+\lambda_{2}\right) \\
& +g\left[1-\frac{g}{(a+c)\left(\frac{b+f}{b e}\right)+\frac{c}{e}-1}-\frac{(a+c)}{b} \cdot \frac{g}{(a+c)\left(\frac{b+f}{b e}\right)+\frac{c}{e}-1}\right]
\end{aligned}
$$

The above equation is the equation analysis process. 